\title{
Major ceRNA regulation and key metabolic signature analysis of intervertebral disc degeneration
}

\author{
Shuai Cao, Jie Li, Kai Yang and Haopeng Li
}

\begin{abstract}
Background and objective: Intervertebral disc degeneration (IDD) is a complex multifactorial and irreversible pathological process. In IDD, multiple competing endogenous RNAs (ceRNA, including mRNA, IncRNA, and pseudogenes) can compete to bind with miRNAs. However, the potential metabolic signatures in nucleus pulposus (NP) cells remain poorly understood. This study investigated key metabolic genes and the ceRNA regulatory mechanisms in the pathogenesis of IDD based on microarray datasets.

Methods: We retrieved and downloaded four independent IDD microarray datasets from the Gene Expression Omnibus. Combining the predicted interactions from online databases (miRcode, miRDB, miRTarBase, and TargetScan), differentially expressed IncRNAs (DEIncRNAs), miRNAs (DEmiRNAs), and mRNAs (DEmRNAs) were identified. A ceRNA network was constructed and annotated using GO and KEGG pathway enrichment analyses. Moreover, we searched the online metabolic gene set and used support vector machine (SVM) to find the critical metabolic DEmRNA(s) and other DERNAs. Differential gene expression was validated with a merged dataset.

Results: A total of 45 DEmRNAs, 36 DElncRNAs, and only one DEmiRNA (miR-338-3p) were identified in the IDD microarray datasets. GO and KEGG pathway enrichment analyses revealed that the DEmRNAs were predominantly enriched in the PI3K-Akt signaling pathway, MAPK signaling pathway, IL-17 signaling pathway, apoptosis, and cellular response to oxidative stress. Based on SVM screening, 6-phosphofructo-2-kinase/fructose-2,6-bisphosphatase (PFK/FBPase) 2 is the critical metabolic gene with lower expression in IDD, and AC063977.6 is the key IncRNA with lower expression in IDD. The ceRNA hypothesis suggests that AC063977.6, miR-338-3p (high expression), and PFKFB2 are dysregulated as an axis in IDD.
\end{abstract}

Conclusions: The results suggest that InCRNA AC063977.6 correlate with PFKFB2, the vital metabolic signature gene, via targeting miR-338-3p during IDD pathogenesis. The current study may shed light on unraveling the pathogenesis of IDD.

Keywords: Intervertebral disc degeneration, Metabolism, Competing endogenous RNA, MicroRNA, Long noncoding RNA

\footnotetext{
*Correspondence: lihaopeng3993@163.com

Department of Orthopedics, The Second Affiliated Hospital of Xi'an Jiaotong

University, Xi'an 710004, China
}

C C The Author(s). 2021 Open Access This article is licensed under a Creative Commons Attribution 4.0 International License, which permits use, sharing, adaptation, distribution and reproduction in any medium or format, as long as you give appropriate credit to the original author(s) and the source, provide a link to the Creative Commons licence, and indicate if changes were made. The images or other third party material in this article are included in the article's Creative Commons licence, unless indicated otherwise in a credit line to the material. If material is not included in the article's Creative Commons licence and your intended use is not permitted by statutory regulation or exceeds the permitted use, you will need to obtain permission directly from the copyright holder. To view a copy of this licence, visit http://creativecommons.org/licenses/by/4.0/ The Creative Commons Public Domain Dedication waiver (http://creativecommons.org/publicdomain/zero/1.0/) applies to the data made available in this article, unless otherwise stated in a credit line to the data. 


\section{Background}

Intervertebral disc degeneration (IDD), a complex multifactorial and age-dependent condition, is a critical contributor to low-back pain (LBP) [1]. The increasing incidence of IDD not only affects the life quality of patients because of chronic pain and disability, but also causes a severe financial burden to the health care system [2]. Moreover, current prevention, diagnosis, and treatment modalities for IDD are hampered due to its ambiguous pathogenic mechanisms. Previous studies have shown that IDD is characterized by the abnormal proliferation of nucleus pulposus (NP) cells, degradation of proteoglycans and collagen in the extracellular matrix (ECM), imbalance of homeostasis, and accelerated transition of NP cells from a healthy state to a catabolic and degenerative state [3]. It is known that the cellular function of all life forms relies closely on metabolism [4, 5]. Metabolic genes are believed to play essential roles in various cellular functions and many diseases such as hepatic fibrosis, central nervous system diseases, and hematologic malignancies [6-8]. However, investigations concerning the role of metabolic networks in IDD remain sparse. Hence, in-depth comprehension of metabolic gene expression and the regulatory mechanisms of noncoding RNA may provide novel insights into the onset, development, and diagnosis of IDD.

Long noncoding RNAs (lncRNAs), a group of RNA molecules consisting of more than 200 nucleotides with no or feeble protein-coding ability [3], have been of great interest because of their ability to regulate gene expression in various pathological and biological processes, such as cell proliferation and apoptosis $[9,10]$. MicroRNAs (miRNAs), consisting of approximately 18-22 nucleotides without coding function, mediate posttranscriptional regulation of target messenger RNA (mRNA) [11]. Ample studies indicate that lncRNAs can act as natural microRNA (miRNA) sponges to bind miRNAs, acting as competing endogenous RNAs (ceRNAs) [12-14]. The ceRNA crosstalk plays a critical role in modulating gene expression [15]. Accumulating evidence has demonstrated the aberrant expression of several mRNAs, miRNAs, and lncRNAs in NP cells in IDD and explained the relationship between them with respect to autophagy, apoptosis, and cell cycle [11, 16, 17]. Nevertheless, metabolic gene-associated ceRNA regulated IDD progression remains largely unstudied. Hence, further exploration of metabolism-related ceRNA crosstalk in NP cells will benefit our understanding of the metabolic gene regulatory network, which is of considerable significance for understanding IDD pathogenic mechanisms. Besides, it is vital to identify the key metabolic gene(s) and relevant key regulated noncoding RNAs in the IDD. In addition, we previously screened core RNAs of IDD based not only on differential expression (DE) but also using the machine learning technology, namely support vector machine (SVM) [18].

Currently, the definitive diagnosis of IDD is sometimes difficult because the intervertebral disc has a particular anatomical structure. In addition, LBP usually has atypical clinical features [19], and the definitive diagnosis is not often achieved using clinical imaging methods [20]. It is even more difficult to correctly diagnose dorsal disc migrations [21], and misdiagnosis often occurs [22]. A transcriptomic signature is beneficial for clear and early diagnosis and timely treatment of patients with IDD [23], especially those that are at a high risk of IDD. Currently, the metabolic gene signature is a collection of metabolism-related genes that can characterize the outcome events; it accounts for the top differentially regulated genes in many disorders but is relatively poorly explored in IDD [24, 25]. In the current study, we aimed to explore the key metabolic gene(s) signature and its key ceRNA regulatory systems to provide insight into IDD pathogenesis. Combining database prediction and gene expression validation, we explored the metabolic abnormalities of NP cells in IDD and revealed their underlying metabolic, molecular signatures and regulation profiles based on microarray datasets.

\section{Methods}

\section{Data acquisition and study design (Fig. 1)}

After searching the National Center of Biotechnology Information (NCBI) Gene Expression Omnibus (GEO) database (https://www.ncbi.nlm.nih.gov/geo), we retrieved and downloaded all four IDD microarray datasets (lncRNA microarray set, GSE56081; miRNA microarray sets GSE116726 and GSE19943; and mRNA microarray sets, GSE56081 and GSE70362). According to the Thompson grading system, grade I to grade III NP samples were classified as control samples, and grade IV and grade V NP samples were classified as IDD samples [26]. GSE56081 consists of five IDD and five control samples, whereas GSE116726 and GSE19943 include three IDD and three control samples, respectively. The GSE70362 consists of 14 control samples and 10 IDD samples (Table 1). In order to obtain standardized data, we performed batch normalization on GSE116726 and GSE19943 using the R package 'sva'. For mRNA analysis, the GSE56081 microarray set was used for initial exploration, and the GSE70362 microarray set was normalized, merged and used for further validation (batch normalization and merging with GSE56081 to obtain large sample size). Because the data were publicly obtained from the GEO database, we strictly followed the publication guidelines approved by GEO (details with platform information, sample size, and access are shown in Supplementary Table 1. The characteristics of IDD patients in related GEO datasets are shown in 


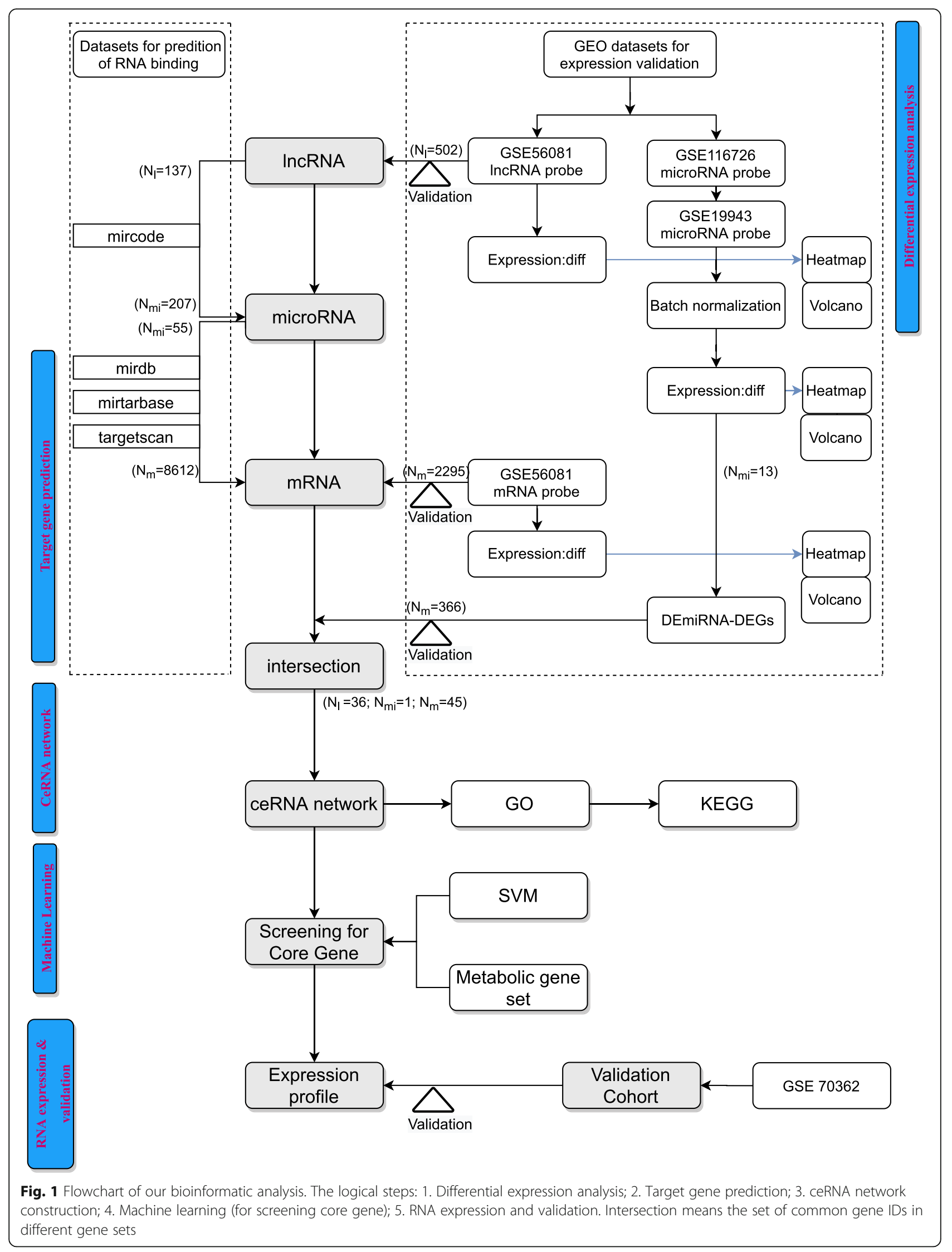


Table 1 Details and sample size with studied GEO datasets

\begin{tabular}{lllll}
\hline Datasets & Platform information & Control(N) & IDD(N) & Application \\
\hline GSE56081 & GPL15314 Arraystar Human LncRNA microarray V2.0 & 5 & 5 & identification of DElncRNA \\
GSE116726 & GPL20712 Agilent-070156 Human miRNA [miRNA version] & 3 & 3 & identification of DEmiRNA \\
GSE19943 & GPL9946 Exiqon human miRCURY LNA ${ }^{\text {TM }}$ miRNA Array V11.0 & 3 & 3 & identification of DEmiRNA \\
GSE56081 & GPL15314 Arraystar Human LncRNA microarray V2.0 & 5 & 5 & identification of metabolic DEGs \\
GSE70362 & GPL17810[HG-U133_Plus_2] Affymetrix Human Genome U133 & 14 & 10 & validation of metabolic DEGs
\end{tabular}

Abbreviations: GEO Gene Expression Omnibus, IDD Intervertebral disc degeneration, DEIncRNAs differentially expressed IncRNAs, DEmiRNAs differentially expressed miRNAs, DEGs differentially expressed genes

Supplementary Table 2). And, ethics committee approval was not required to conduct this study.

\section{DE IncRNAs, miRNAs, mRNA screening}

The ceRNA network was constructed based on DE analysis for differentially expressed lncRNAs (DElncRNAs), differentially expressed miRNAs (DEmiRNAs) and differentially expressed mRNAs [DEmRNAs, responding to differentially expressed genes (DEGs)] of microarrays with $\mathrm{R}$ package 'limma' and the prediction for interactions in the online database. First, DElncRNAs were identified between IDD and control samples in GSE56081. Next, a highly conservative miRNA family profile on the Mircode database (http://www.mircode.org/) was used to predict interactions between miRNAs for the above DElncRNAs. To validate and ensure the reliability and accuracy of the predicted data, we obtained another set of DEmiRNAs in IDD samples from GSE116726 and GSE19943 for comparison, with the thresholds of $P<0.05$ (two-tailed) and $\mid \log _{2}$ fold change (FC) $\mid \geq 1$. Then, the overlapped miRNAs were used for the final DElncRNA screening.

\section{Prediction of DEmRNAs}

As shown in Fig. 2, DEmiRNA targets were predicted using three independent experimental databases, including miRDB (http://mirdb.org/), miRTarBase (http:// mirtarbase.mbc.nctu.edu.tw/php/index.php), and TargetScan (http://www.targetscan.org/vert_72/). Here, a

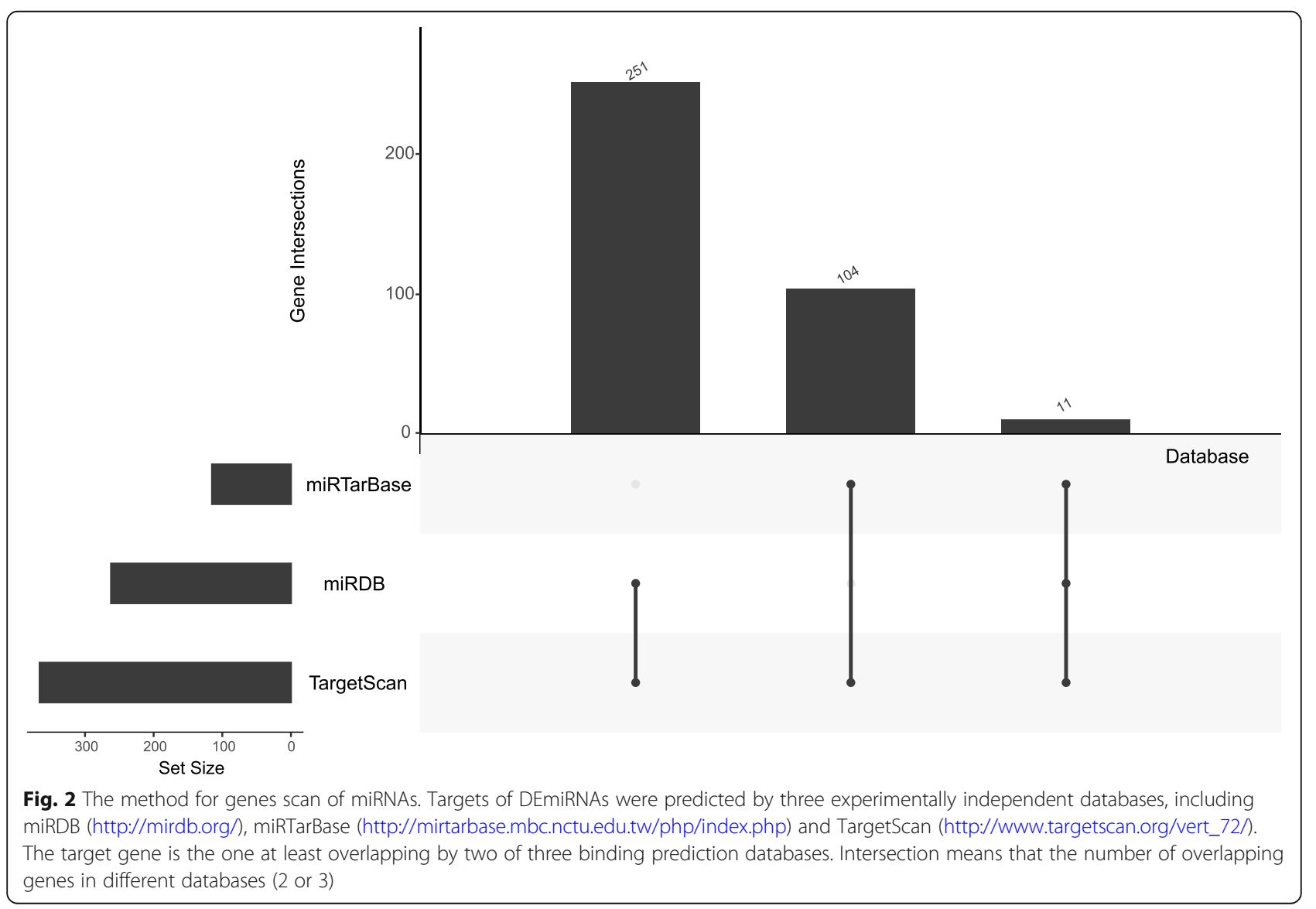


scoring system was used to predict genes in the DEmRNAs group (DEmRNAs-1). One score was added to the target (mRNA) for each above database that could interact with each other. If the score was $\geq 2$, the mRNA was put into the DEmRNAs-1 group. Meanwhile, the DEmRNAs-2 group was directly identified in GSE56081. Besides, the targets of the overlapped miRNAs from datasets GSE116726 and GSE19943 were predicted as the DEmRNAs-3 group. Next, we kept the overlapped mRNAs in all DEmRNA groups (DEmRNAs-1, DEmRNAs-2, and DEmRNAs-3) as the final DEmRNAs in this study.

After the DE analysis process (Fig. 1), we visualized DElncRNAs, DEmiRNAs, and DEGs between IDD and control samples using clustering heat maps and volcano maps, respectively, using the $\mathrm{R}$ package 'pheatmap'. The key DElncRNAs, DEmiRNAs, and DEGs were selected as the sources of the ceRNA network, which was constructed using Cytoscape3.8.0 [27]. Dark blue rectangle nodes represent the lncRNAs in the network, the purple rhombus nodes represent the target genes, and lines indicate interactions.

\section{Functional enrichment analyses of the ceRNA network}

We performed Gene Ontology (GO) and Kyoto Encyclopedia of Genes and Genomes (KEGG) pathway enrichment analyses of DEGs using R packages ("clusterProfiler", "org. Hs.eg.db", "enrichplot", and "ggplot2" package) with identified filters of $p$-Value $<0.05$ and qValue $<0.05[28-30]$.

\section{Feature selection based on SVM and metabolic gene set}

Support vector machine (SVM), a machine learning technology, is a supervised learning model with associated learning algorithms that analyzes data used for classification and regression analyses. Based on the expression levels of the ceRNA, we used the R package 'caret' and adopted 5-fold cross-validation to conduct the SVM model for screening vital RNAs. The random seed was set to 39 in all SVM progress. Moreover, we searched for genes on the metabolic gene set based on metabolism-related pathways, which were downloaded from the gene set enrichment analysis (GSEA) website (GSEA, c2.cp.kegg.v7.1.symbols.gmt; https://www.gseamsigdb.org/gsea/downloads.jsp). We obtained 948 metabolism-related genes for follow-up analysis (Supplementary Table 3). Finally, we used the Venn method (http://bioinformatics.psb.ugent.be/webtools/Venn/) to search for the key DEG(s) from the results of SVM, expression levels, and the metabolic gene set. Furthermore, we searched for other differentially expressed RNAs (DERNAs) based on the SVM and expression levels to find the critical ceRNAs regulated by the key metabolic gene(s).

\section{Statistical analyses}

The $\mathrm{R}$ software (version 3.6.2, The $\mathrm{R}$ Foundation for Statistical Computing, Vienna, Austria) and MedCalc statistical software (Version 19.0.4, MedCalc Software bvba, Ostend, Belgium) were used for the statistical analyses. Prism (version 8.0, GraphPad Prism Inc.) and $\mathrm{R}$ software were used for graphics. DElncRNAs, DEmiRNAs, and DEmRNAs between IDD and control samples were identified using filters with $P<0.05$ (two-tailed) and $\left|\log _{2} \mathrm{FC}\right| \geq 1$. A positive $\log _{2} \mathrm{FC}$ value indicated upregulated expression, whereas a negative $\log _{2} \mathrm{FC}$ value indicated downregulated expression. The chi-square test was used for enrichment analyses. Difference with $P<$ 0.05 were considered statistically significant.

\section{Results}

Identification of DEIncRNAs, DEmiRNAs, and DEGs using integrated microarray analysis

A total of 502 DElncRNAs and 2295 DEmRNAs were directly identified on GSE56081. Moreover, 15 DEmiRNAs were identified in GSE116726 and GSE19943 (Figs. 1 and 3).

\section{Target prediction for DElncRNAs, DEmiRNA, and DEGs}

Of the 502 DElncRNAs, 137 could bind to 207 miRNAs. Target prediction for the above 207 miRNAs revealed that 55 miRNAs could predict the downstream target mRNAs (DEmRNAs-1 group). Surprisingly, we found that miR-338-3p was the only overlapping miRNA between the 55 miRNAs and the 15 DEmiRNAs. Thus, focusing on miR-338-3p, we mined 36 upstream IncRNAs that could bind to it. Furthermore, 366 downstream mRNAs (DEmRNAs-3 group) related to miR-338-3p were also predicted. There were 8612 target mRNAs (DEmRNAs-1 group) of the 55 miRNAs, which were predicted using the miRDB, miRTarBase, and TargetScan databases (Fig. 2). Finally, 45 DEmRNAs (final DEmRNAs, Supplementary Table 4), which bind to miR338-3p, were the overlapping genes among the 366 mRNAs, 8612 mRNAs, and 2295mRNAs. The 45 DEmRNAs, which combine with miR-338-3p, were concentrated.

To reveal the underlying molecular signatures of IDD, we constructed a ceRNA network using overlapped DElncRNAs and DEGs, the core of which was miR-3383p. The ceRNA network displayed 45 miR-338-3p/ lncRNA links and 36 miR-338-3p/mRNA links (Fig. 4a).

\section{Functional enrichment analyses}

The GO terms (45 DEGs) indicated that several genes, which existed in the cell trailing edge, were enriched in the phosphatidylinositol 3-kinase signaling pathway and the cellular response to reactive oxidative stress in biological process (Fig. 4b, details of actual gene IDs and 

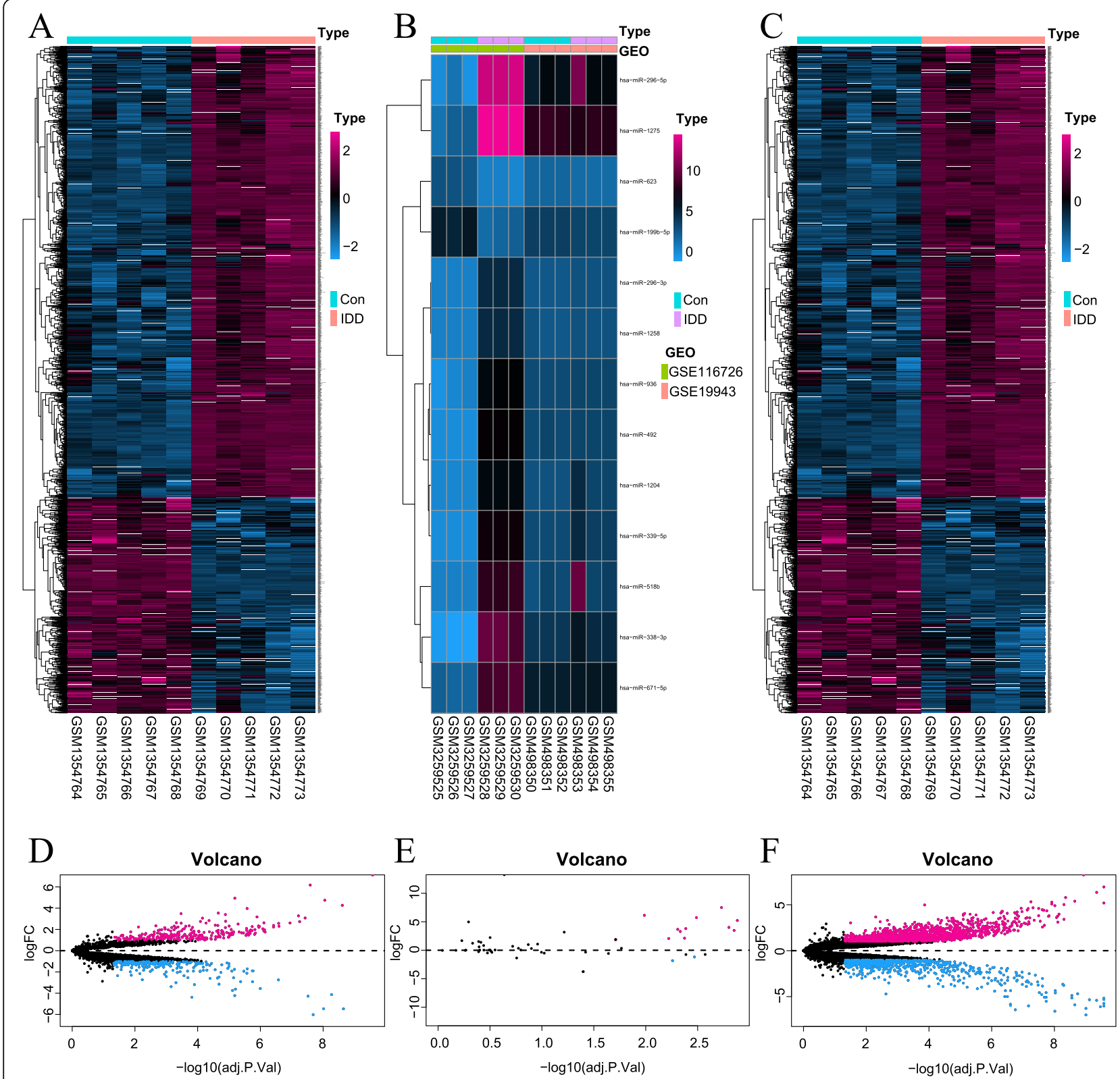

Fig. 3 Difference analysis. a-c The differentially expressed heat-maps of IncRNA, microRNA, and mRNA. The IncRNA heat-map of GSE56081 dataset (a); The merged microRNA heat-maps of GSE1 16726 and GSE19943 datasets (b); The mRNA heat-map of GSE56081 dataset. d-f The volcano plot (c). The IncRNA dataset of GSE56081 (d); The merged microRNA datasets of GSE116726 and GSE19943 (e); The mRNA dataset of GSE56081 (f). The screening condition: absolute values of $\log$ (fold change) $>1.0$ and adjusted $p$-Value $<0.05$. In the plots, the rose red and blue dots represent high and low RNA expressions with statistical significance between the IDD NPs and the normal NPs, respectively. And, the black dots represent no statistical significance between them

GO descriptions are shown in Supplementary Table 5). Moreover, the KEGG pathway enrichment analysis showed that the 45 DEGs were predominantly enriched in 16 pathways, including the MAPK signaling pathway, IL-17 signaling pathway, apoptosis, and TNF signaling pathway, et al. (Fig. 4c) The details of actual gene IDs per pathway are shown in Supplementary Table 6.
Feature selection based on SVM and metabolic gene set In dataset GSE56081, we defined 45 overlapping DEGs. Moreover, the metabolic gene set consisted of 948 genes. Hence, we used the SVM method to build a classifier and create a decision boundary for the 36 DElncRNAs and 45 DEGs (Fig. 5a, b). SVM was not used for screening DEmiRNAs because miR-338-3p was the only core 


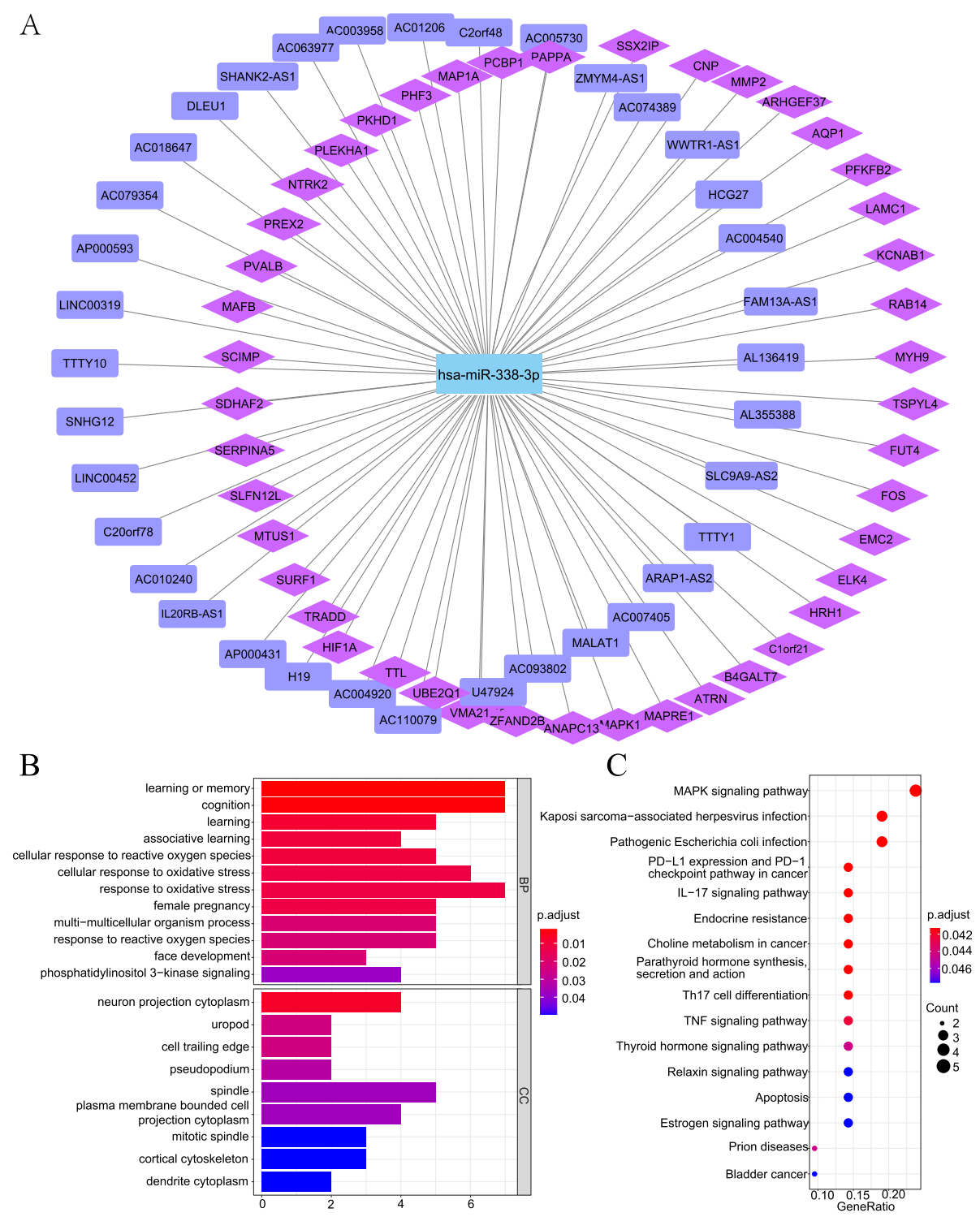

Fig. 4 The ceRNA network and its enrichment analysis by GO annotation and pathways identified by KEGG for Differentially expressed genes. The ceRNA network created by Cytoscape 3.8.3 (a). The connections DEIncRNA (purple rectangle), DEmicroRNA (blue), and target genes (purple pink rhombus) were represented as nodes, and their interactions were denoted by lines. The top terms of enriched GO analysis (b). The top terms of enriched KEGG pathway [28-30] (c). Abbreviation: GO, the gene ontology; KEGG, the Kyoto encyclopedia of genes and genomes; BP, the biological process; CC, the cellular component. The details for actual gene IDs and GO descriptions were shown in Supplementary Table 5. The details for actual gene IDs per KEGG pathway were shown in Supplementary Table 6. We are grateful to Kanehisa Laboratories

DEmiRNA. Then, the Venn method was used to search for the key DElncRNAs and DEGs from the results of SVM signature, expression levels, and the metabolic gene set (Fig. 5c, d, e). The core DElncRNA was lncRNA AC063977.6 (Fig. 5c). The core metabolic gene was 6phosphofructo-2-kinase/fructose-2,6-bisphosphatase 2 (PFK/FBPase2) after training by SVM and intersecting with the metabolic gene set (Fig. 5e). The expression levels of core RNAs are presented as a histogram (Fig. 5f).

\section{Discussion}

The metabolic homeostasis and the construction of the ECM of intervertebral discs mainly depend on its active NP cells, which form the inner core of the intervertebral disc. An imbalance of cellular functions because of metabolic imbalance, nutrient deprivation, dysregulated apoptosis, and some transcellular signaling interrupts homeostasis in NP cells, resulting in IDD [31-33]. Furthermore, the metabolic signature represents a broad molecular signature of systemic metabolism and covers 


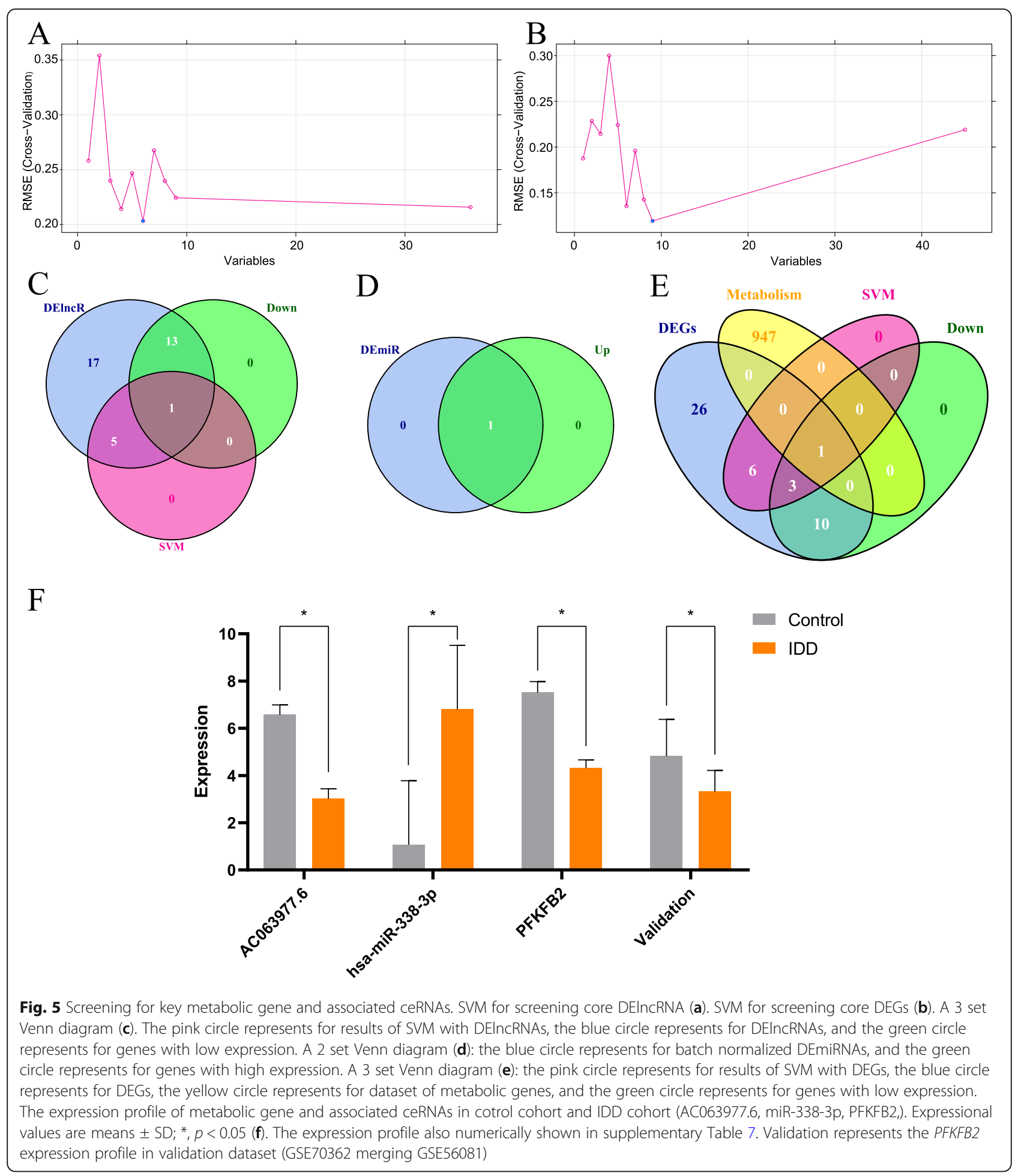

multiple cellular metabolic pathways [34]. The metabolic signature and its relevant mechanism are critical for understanding the cellular mechanisms of various diseases. Accordingly, it would be reasonable to assume that NP cell metabolic disorder might be a pathogenic factor of IDD. However, the metabolic investigations and associated ceRNA regulation mechanism of NP cells in IDD remaine inconclusive. The ceRNAs regulate the expression of various genes and play a significant role in the pathogenesis of many diseases [35]. Identifying ceRNA regulation with the metabolic signatures in NP cells would shed a novel light on IDD. Thus, based on 
the GEO dataset, we identified a novel gene metabolic signature and related ceRNA regulatory mechanisms to gain insight into the IDD pathogenic mechanism.

In the present study, 45 DEGs and 36 DElncRNAs were identified. MiR-338-3p was the only key DEmiRNA, which was upregulated in IDD. A vast ceRNA network was constructed to reveal the underlying molecular signatures of IDD. GO annotation revealed that the DEmRNAs/DEGs were mostly enriched in the PI3K-Akt signaling pathway. The PI3K-Akt signaling pathway participates in the synthesis of ECM, regulating apoptosis and cell proliferation in IDD. Furthermore, the GO annotation showed that many biological processes were categorized in response to oxidative stress of NP cells, which indicates that oxygen and oxygen-associated processes might play an essential role in IDD. The KEGG pathway enrichment analysis showed that the MAPK signaling pathway, IL-17 signaling pathway, apoptosis, and TNF signaling pathway might be related to IDD. Based on batch normalization, we found that miR-338-3p was the only DEmiRNA validated in two datasets and was correctly predicted for lncRNAmiRNA and mRNA-miRNA binding. However, there were 45 overlapping DEGs and 948 genes defined by the metabolic gene set. To further determine the key metabolic signature and critical lncRNA(s) of the 36 DElncRNAs in IDD, we used the SVM method. Thus, AC063977.6 was identified as the critical lncRNA with recursive feature elimination, based on the results of SVM and related expression regulation of DElncRNAs. Moreover, $P F K F B 2$ is the key metabolic gene with recursive feature elimination, based on the results of SVM, metabolic gene set, and related expression regulation of DEmRNAs.

According to the online database prediction, AC063977.6 directly targets miR-338-3p, whereas miR338-3p directly targets PFKFB2, suggesting that AC063977.6, miR-338-3p, and PFKFB2 might form an axis that modulates NP cell metabolism in IDD. Furthermore, the gene expression validation based on microarray data of GSE116726 and GSE19943 further enhanced the credibility of this axis. In conclusion, we found that AC063977.6, miR-338-3p, and PFKFB2 formed an axis in IDD to modulate the metabolism of NP cells. This study revealed that miR-338-3p was upregulated in degenerating NP cells, accompanied by downregulation of PFKFB2 expression. Moreover, AC063977.6, acting as a ceRNA sponging miR-338-3p, was significantly attenuated in IDD progression.

The central part of the DEGs is PFKFB2, which is an isoform of the PFKFB family that mainly regulates glycolytic metabolism and catalyzes the fructose 2,6bisphosphate (Fru-2,6-P) [36]. PFKFB2 is a key bifunctional enzyme involved in glycolytic metabolism
[37]. Moreover, glycolysis significantly diminishes cellular oxidative stress $[38,39]$. Similarly, bioinformatics analysis with the GEO microarray datasets revealed that the DEGs were significantly enriched in the oxidative stress process in $\mathrm{GO}$ enrichment. Compared to normal cells, several cancer cells exhibit elevated expression levels of $P F K F B$ [40]. However, in this study, we found that the expression level of PFKFB2 in NP cells with IDD was lower than that in control NP cells, suggesting that reduced glycolysis may increase the level of cellular oxidative stress in NP cells; thus, it may be associated with IDD.

Accumulating evidence suggests that lncRNAs and miRNAs dysregulate essential pathological processes of IDD, such as apoptosis, angiogenesis, ECM degradation, and inflammatory responses [41]. The miR-338$3 p$ originates from the apoptosis-associated tyrosine kinase gene, and it is dysregulated in many tumors and plays distinct roles in different diseases [42]. The upregulation of miR-338-3p could promote glioma cell invasion and metastasis of lung cancer, whereas the downregulation of miR-338-3p was related to poor outcomes in gastric cancer [43-45]. In this study, miR-338-3p was remarkably upregulated in IDD, serving as an impetus with metabolic dysregulation by targeting PFKFB2, thereby disturbing the metabolism of NP cells. IDD severely threatens the health of patients, and identification of genetic signature is beneficial for its early diagnosis and early treatment [23]. Moreover, a strong negative correlation was observed between IDD and PFKFB2 expression levels in both the training cohort and validation cohort, which indicates that low expression of PFKFB2 is a key factor in IDD. The results suggest that PFKFB2 is an player in IDD. According to our experience in clinical work and reported literature, misdiagnosis of IDD occasionally occurs, which often leads to incorrect treatment and even unnecessary surgery. Based on our results, the metabolic signature could be used in providing clues for IDD mechanism research, and may aid in surgery for differential diagnosis in clinical practice.

\section{Limitations of this study}

There are some limitations to this study. First, we focused only on the regulatory roles of target genes without further classification into specific subgroups according to their functions, which limited the excavation of those data. Second, further in vitro and in vivo experiments on the sophisticated regulating mechanism involved with AC063977.6 and miR-338 in IDD progression are urgently needed. Third, although a small sample size is a common problem in orthopedic research, it is undeniable that this study is based on a small sample size (a total of 46 samples 
were included). Sufficient in vitro and in vivo experiments are warranted to establish the functions and mechanisms of the lncRNAs and miRNAs involved in the pathogenesis of IDD and may further promote the translation of precise gene diagnosis in IDD.

\section{Conclusion}

The results suggest that lncRNA AC063977.6, acting as a ceRNA that sponges miR-338, correlating with the metabolism of NP cells via the AC063977.6/miR-338/ PFKFB2 axis in IDD. PFKFB2 can serve as a metabolic biomarker to contribute to the differential diagnosis of IDD in clinical practice.

\begin{abstract}
Abbreviations
IDD: Intervertebral disc degeneration; miRNAs: MicroRNAs; IncRNAs: Long noncoding RNAs; ceRNA: Competing endogenous RNA; NP: Nucleus pulposus; GEO: Gene Expression Omnibus; DE: Differential expression; DEIncRNAs: Differentially expressed IncRNAs; DEmiRNAs: Differentially expressed miRNAs; DEmRNAs: Differentially expressed mRNAs; SVM: Support vector machine; PFKFB: 6-phosphofructo-2-kinase/fructose-2,6-bisphosphatas; AUC: The area under curve; LBP: Low-back pain; ECM: Extracellular matrix; mRNA: Messenger RNA; DEGs: Differentially expressed genes; GO: Gene Ontology; KEGG: Kyoto Encyclopedia of Genes and Genomes; ROC: Receiver operating characteristic
\end{abstract}

\section{Supplementary Information}

The online version contains supplementary material available at https://doi. org/10.1186/s12891-021-04109-8.

Additional file 1: Table S1. Details with platform information, sample size, and access in studied GEO datasets. Table S2. The characteristics of IDD patients in related datasets. Table S3. The 948 metabolism-related genes/ The metabolic gene set. Table S4. The final 45 DEmRNAs (or DEGs). Table S5. The details for actual gene IDs and GO descriptions. Table S6. The details for actual gene IDs per pathway. Table S7. Expression profile.

\section{Acknowledgments}

Not applicable.

\section{Authors' contributions}

Shuai Cao collected the cases and drafted the manuscript. Shuai Cao and Jie Li analyzed the data. Haopeng Li and Shuai Cao designed the study. All authors read and approved the final manuscript.

\section{Funding}

Not applicable.

\section{Availability of data and materials}

The datasets generated during the current study are available in the GEO repository [https://www.ncbi.nlm.nih.gov/geo]. The datasets used and/or analyzed during the current study are also available from the corresponding author upon reasonable request. The authors declare that the databases described in the manuscript are available for testing. Details pertaining to the dataset platform information, sample size, and access are shown in Supplementary Table 1.

\section{Declarations}

Ethics approval and consent to participate Not applicable.

\section{Consent for publication}

Not applicable.

\section{Competing interests}

The authors declare that they do not have anything to disclose regarding conflict of interest with respect to this manuscript.

Received: 17 October 2020 Accepted: 22 February 2021

Published online: 06 March 2021

\section{References}

1. Risbud MV, Shapiro IM. Role of cytokines in intervertebral disc degeneration: pain and disc content. Nat Rev Rheumatol. 2014;10(1):44-56.

2. Capoor MN, Ruzicka F, Machackova T, Jancalek R, Smrcka M, Schmitz JE, Hermanova M, Sana J, Michu E, Baird JC, et al. Prevalence of Propionibacterium acnes in intervertebral discs of patients undergoing lumbar microdiscectomy: a prospective cross-sectional study. PLoS One. 2016;11(8):e0161676.

3. Zhao K, Zhang Y, Yuan H, Zhao M, Zhao D. Long noncoding RNA LINC00958 accelerates the proliferation and matrix degradation of the nucleus pulposus by regulating miR-203/SMAD3. Aging. 2019;11(23):1081425.

4. Jensen M, Röthlisberger U, Rovira C. Hydroxide and proton migration in aquaporins. Biophys J. 2005;89(3):1744-59.

5. Leveillard T, Philp NJ, Sennlaub F. Is Retinal Metabolic Dysfunction at the Center of the Pathogenesis of Age-related Macular Degeneration? Int J Mol Sci. 2019;20(3)

6. Allegra A, Innao V, Gerace D, Bianco O, Musolino C. The metabolomic signature of hematologic malignancies. Leuk Res. 2016;49:22-35.

7. Chang ML, Yang SS. Metabolic Signature of Hepatic Fibrosis: From Individual Pathways to Systems Biology. Cells. 2019;8(11).

8. Dumas ME, Davidovic L. Metabolic profiling and Phenotyping of central nervous system diseases: metabolites bring insights into brain dysfunctions. J Neurolmmune Pharmacol. 2015;10(3):402-24.

9. Li P, Xue WJ, Feng Y, Mao QS. Long non-coding RNA CASC2 suppresses the proliferation of gastric cancer cells by regulating the MAPK signaling pathway. Am J Transl Res. 2016;8(8):3522-9.

10. Zhao W, Geng D, Li S, Chen Z, Sun M. LncRNA HOTAIR influences cell growth, migration, invasion, and apoptosis via the miR-20a-5p/HMGA2 axis in breast cancer. Cancer Med. 2018;7(3):842-55.

11. Zhan S, Wang K, Xiang Q, Song Y, Li S, Liang H, Luo R, Wang B, Liao Z, Zhang $Y$, et al. IncRNA HOTAIR upregulates autophagy to promote apoptosis and senescence of nucleus pulposus cells. J Cell Physiol. 2020; 235(3):2195-208

12. Ma F, Wang SH, Cai Q, Jin LY, Zhou D, Ding J, Quan ZW. Long non-coding RNA TUG1 promotes cell proliferation and metastasis by negatively regulating miR-300 in gallbladder carcinoma. Biomed Pharmacother. 2017; 88:863-9.

13. Tay Y, Rinn J, Pandolfi PP. The multilayered complexity of ceRNA crosstalk and competition. Nature. 2014;505(7483):344-52.

14. Zhang CZ. Long non-coding RNA FTH1P3 facilitates oral squamous cell carcinoma progression by acting as a molecular sponge of miR-224-5p to modulate fizzled 5 expression. Gene. 2017;607:47-55.

15. Smillie CL, Sirey T, Ponting CP. Complexities of post-transcriptional regulation and the modeling of ceRNA crosstalk. Crit Rev Biochem Mol Biol. 2018;53(3):231-45

16. Han Z, Wang J, Gao L, Wang Q, Wu J. Aberrantly expressed messenger RNAs and long noncoding RNAs in degenerative nucleus pulposus cells cocultured with adipose-derived mesenchymal stem cells. Arthritis Res Ther. 2018;20(1):182.

17. Xi Y, Jiang $T$, Wang $W, Y u$ J, Wang $Y$, Wu X, He $Y$. Long non-coding HCG18 promotes intervertebral disc degeneration by sponging miR-146a-5p and regulating TRAF6 expression. Sci Rep. 2017;7(1):13234.

18. Sanz H, Valim C, Vegas E, Oller JM, Reverter F. SVM-RFE: selection and visualization of the most relevant features through non-linear kernels. BMC Bioinformatics. 2018;19(1):432.

19. Xu JX, Yang SD, Wang BL, Yang DL, Ding WY, Shen Y. Correlative analyses of isolated upper lumbar disc herniation and adjacent wedge-shaped vertebrae. Int J Clin Exp Med. 2015;8(1):1150-5.

20. Izzo R, Popolizio T, D'Aprile P, Muto M. Spinal pain. Eur J Radiol. 2015;84(5): 746-56.

21. Zarrabian MM, Diehn FE, Kotsenas AL, Wald JT, Yu E, Nassr A. Dorsal lumbar disc migrations with lateral and ventral epidural extension on axial MRI: a 
case series and review of the literature. AJNR Am J Neuroradiol. 2016;37(11): 2171-7

22. Ghaly RF, Perciuleac Z, Candido KD, Knezevic NN. Athletic pubalgia misdiagnosed as lumbar radiculopathy - a case report. Surg Neurol Int. 2019;10:224

23. Feng $Y$, Egan $B$, Wang J. Genetic factors in intervertebral disc degeneration. Genes Dis. 2016;3(3):178-85.

24. Wu ZH, Tang Y, Zhou Y. A Metabolic Gene Signature to Predict Overal Survival in Head and Neck Squamous Cell Carcinoma. Mediat Inflamm 2020;2020:6716908.

25. Zeng F, Su J, Peng C, Liao M, Zhao S, Guo Y, Chen X, Deng G. Prognostic implications of metabolism related gene signature in cutaneous melanoma. Front Oncol. 2020;10:1710.

26. Gruber HE, Hoelscher GL, Ingram JA, Hanley EN Jr. Genome-wide analysis of pain-, nerve- and neurotrophin -related gene expression in the degenerating human annulus. Mol Pain. 2012;8:63.

27. Shannon P, Markiel A, Ozier O, Baliga NS, Wang JT, Ramage D, Amin N, Schwikowski B, Ideker T. Cytoscape: a software environment for integrated models of biomolecular interaction networks. Genome Res. 2003;13(11): 2498-504.

28. Kanehisa M. Toward understanding the origin and evolution of cellular organisms. Protein Sci. 2019;28(11):1947-51.

29. Kanehisa M, Furumichi M, Sato Y, Ishiguro-Watanabe M, Tanabe M. KEGG: integrating viruses and cellular organisms. Nucleic Acids Res. 2021;49(D1): D545-d551.

30. Kanehisa M, Goto S. KEGG: Kyoto encyclopedia of genes and genomes. Nucleic Acids Res. 2000;28(1):27-30

31. Chen D, Xia D, Pan Z, Xu D, Zhou Y, Wu Y, Cai N, Tang Q, Wang C, Yan M, et al. Metformin protects against apoptosis and senescence in nucleus pulposus cells and ameliorates disc degeneration in vivo. Cell Death Dis. 2016;7(10):e2441.

32. Liu J, Yuan C, Pu L, Wang J. Nutrient deprivation induces apoptosis of nucleus pulposus cells via activation of the BNIP3/AIF signalling pathway. Mol Med Rep. 2017;16(5):7253-60

33. Liao Z, Luo R, Li G, Song Y, Zhan S, Zhao K, Hua W, Zhang Y, Wu X, Yang C. Exosomes from mesenchymal stem cells modulate endoplasmic reticulum stress to protect against nucleus pulposus cell death and ameliorate intervertebral disc degeneration in vivo. Theranostics. 2019;9(14):4084-100.

34. Park HJ, Kim S, Li W. Model-based analysis of competing-endogenous pathways (MACPath) in human cancers. PLoS Comput Biol. 2018;14(3): e1006074

35. Yan J, Song J, Qiao M, Zhao X, Li R, Jiao J, Sun Q. Long noncoding RNA expression profile and functional analysis in psoriasis. Mol Med Rep. 2019; 19(5):3421-30

36. Wang X, Li D, Wu H, Liu F, Liu F, Zhang Q, Li J. LncRNA TRPC7-AS1 regulates nucleus pulposus cellular senescence and ECM synthesis via competing with HPN for miR-4769-5p binding. Mech Ageing Dev. 2020;190:111293.

37. Barik S. An intronic microRNA silences genes that are functionally antagonistic to its host gene. Nucleic Acids Res. 2008;36(16):5232-41.

38. Chen $X$, Wei $L$, Zhao S. miR-338 inhibits the metastasis of lung cancer by targeting integrin beta3. Oncol Rep. 2016;36(3):1467-74.

39. Li Y, Huang Y, Qi Z, Sun T, Zhou Y. MiR-338-5p promotes Glioma cell invasion by regulating TSHZ3 and MMP2. Cell Mol Neurobiol. 2018;38(3): 669-77

40. Liu S, Suo J, Wang C, Sun X, Wang D, He L, Zhang Y, Li W. Downregulation of tissue miR-338-3p predicts unfavorable prognosis of gastric cancer. Cancer Biomark. 2017;21(1):117-22.

41. Arden C, Hampson LJ, Huang GC, Shaw JA, Aldibbiat A, Holliman G, Manas D, Khan S, Lange AJ, Agius L. A role for PFK-2/FBPase-2, as distinct from fructose 2,6-bisphosphate, in regulation of insulin secretion in pancreatic beta-cells. Biochem J. 2008;411(1):41-51.

42. Schmidt S, Rainer J, Riml S, Ploner C, Jesacher S, Achmuller C, Presul E, Skvortsov S, Crazzolara R, Fiegl M, et al. Identification of glucocorticoidresponse genes in children with acute lymphoblastic leukemia. Blood. 2006; 107(5):2061-9.

43. Brand KA, Hermfisse U. Aerobic glycolysis by proliferating cells: a protective strategy against reactive oxygen species. FASEB J. 1997;11(5):388-95

44. Poljsak B, Kovac V, Dahmane R, Levec T, Starc A. Cancer etiology: a metabolic disease originating from Life's major evolutionary transition? Oxidative Med Cell Longev. 2019;2019:7831952.
45. Pegoraro C, Maczkowiak F, Monsoro-Burq AH. Pfkfb (6-phosphofructo-2kinase/fructose-2,6-bisphosphatase) isoforms display a tissue-specific and dynamic expression during Xenopus laevis development. Gene Expr Patterns. 2013;13(7):203-11.

\section{Publisher's Note}

Springer Nature remains neutral with regard to jurisdictional claims in published maps and institutional affiliations.
Ready to submit your research? Choose BMC and benefit from:

- fast, convenient online submission

- thorough peer review by experienced researchers in your field

- rapid publication on acceptance

- support for research data, including large and complex data types

- gold Open Access which fosters wider collaboration and increased citations

- maximum visibility for your research: over $100 \mathrm{M}$ website views per year

At BMC, research is always in progress.

Learn more biomedcentral.com/submissions 